\title{
Existence and multiplicity of positive solutions for nonhomogeneous boundary value problems with fractional $q$-derivatives
}

\author{
Yulin Zhao ${ }^{1 *}$, Haibo Chen ${ }^{2}$ and Qiming Zhang ${ }^{1}$
}

${ }^{*}$ Correspondence:

zhaoylch@sina.com

'School of Science, Hunan

University of Technology, Zhuzhou, 412007, China

Full list of author information is

available at the end of the article

\begin{abstract}
In this paper, we study a class of fractional q-difference equations with nonhomogeneous boundary conditions. By applying the classical tools from functional analysis, sufficient conditions for the existence of single and multiple positive solutions to the boundary value problem are obtained in term of the explicit intervals for the nonhomogeneous term. In addition, some examples to illustrate our results are given.

MSC: $34 \mathrm{~A} 08 ; 34 \mathrm{~B} 18 ; 39 \mathrm{~A} 13$
\end{abstract}

Keywords: fractional q-difference equation; nonhomogeneous boundary value problem; positive solution; multiplicity

\section{Introduction}

Fractional differential equations have attracted considerable interest because of its demonstrated applications in various fields of science and engineering including fluid flow, rheology, diffusive transport akin to diffusion, electrical networks, probability [1, 2]. Many researchers have studied the existence of solutions (or positive solutions) to fractional boundary value problems; for example, see [3-10] and the references therein.

The early work on q-difference calculus or quantum calculus dates back to Jackson's papers [11], basic definitions and properties of quantum calculus can be found in the book [12]. For some recent existence results on $q$-difference equations, we refer to [13-15] and the references therein.

The fractional $q$-difference calculus had its origin in the works by Al-Salam [16] and Agarwal [17]. More recently, there seems to be new interest in the study of this subject and many new developments were made in this theory of fractional $q$-difference calculus [1822]. Specifically, fractional $q$-difference equations have attracted the attentions of several researchers. Some recent work on the existence theory of fractional $q$-difference equations can be found in [20,23-31]. However, the study of boundary value problems for nonlinear fractional $q$-difference equations is still in the initial stage and many aspects of this topic need to be explored.

o 2013 Zhao et al: licensee Springer. This is an Open Access article distributed under the terms of the Creative Commons Attribution License (http://creativecommons.org/licenses/by/2.0), which permits unrestricted use, distribution, and reproduction in any medium, provided the original work is properly cited. 
By using a fixed-point theorem in a cone, M. El-Shahed and F. Al-Askar [25] were concerned with the existence of positive solutions to nonlinear $q$-difference equation:

$$
\left\{\begin{array}{l}
{ }_{C} D_{q}^{\alpha} u(t)+a(t) f(u(t))=0, \quad 0<t<1,2<\alpha \leq 3 \\
u(0)=D_{q}^{2} u(0)=0, \quad a D_{q} u(1)+b D_{q}^{2} u(1)=0
\end{array}\right.
$$

where $a, b \geq 0$ and ${ }_{C} D_{q}^{\alpha}$ is the fractional $q$-derivatives of the Caputo type.

In [27], Graef and Kong investigated the boundary value problem with fractional $q$ derivatives

$$
\left\{\begin{array}{l}
\left(D_{q}^{\alpha} u\right)(t)+f(t, u(t))=0, \quad 0<t<1, n-1<\alpha \leq n, n \in \mathbf{N}, \\
\left(D_{q}^{i} u\right)(0)=0, \quad i=0, \ldots, n-2, \quad b D_{q} u(1)=\sum_{j=1}^{m} a_{j} D_{q} u\left(t_{j}\right)+\lambda,
\end{array}\right.
$$

where $\lambda \geq 0$ is a parameter, and the uniqueness, existence and nonexistence of positive solutions are considered in terms of different ranges of $\lambda$.

By applying the Banach contraction principle, Krasnoselskii's fixed-point theorem, and the Leray-Schauder nonlinear alternative, Ahmad, Ntouyas and Purnaras [29] studied the existence of solution for the following nonlinear fractional $q$-difference equation with nonlocal boundary conditions:

$$
\left\{\begin{array}{l}
\left({ }_{C} D_{q}^{\alpha} u\right)(t)=f(t, u(t)), \quad 0 \leq t \leq 1,1<\alpha \leq 2, \\
a_{1} u(0)-b_{1} D_{q} u(0)=c_{1} u\left(\eta_{1}\right), \quad a_{2} u(1)+b_{2} D_{q} u(1)=c_{2} u\left(\eta_{2}\right),
\end{array}\right.
$$

where ${ }_{C} D_{q}^{\alpha}$ is the fractional $q$-derivative of the Caputo type, and $a_{i}, b_{i}, c_{i}, \eta_{i} \in \mathbf{R}$.

Recently, in [32], the authors investigate the following singular semipositone integral boundary value problem for fractional $q$-derivatives equation:

$$
\left\{\begin{array}{l}
\left(D_{q}^{\alpha} u\right)(t)+f(t, u(t))=0, \quad t \in(0,1), 2<\alpha \leq 3 \\
u(0)=\left(D_{q} u\right)(0)=0, \quad u(1)=\mu \int_{0}^{1} u(s) d_{q} s
\end{array}\right.
$$

where $0<\mu<[\alpha]_{q}, D_{q}^{\alpha}$ is the $q$-derivative of Riemann-Liouville type of order $\alpha, f:[0,1] \times$ $(0,+\infty) \rightarrow(-\infty,+\infty)$ is continuous and semipositone, and may be singular at $u=0$.

Since finding positive solutions of boundary value problems is interest in various fields of sciences, fractional $q$-calculus equations has tremendous potential for applications. In this paper, we will deal with the following nonhomogeneous boundary value problem with fractional $q$-derivatives:

$$
\begin{cases}\left(D_{q}^{\alpha} u\right)(t)+f(t, u(t))=0, & t \in(0,1), \\ u(0)=\left(D_{q} u\right)(0)=0, & \gamma\left(D_{q} u\right)(1)+\beta\left(D_{q}^{2} u\right)(1)=\lambda,\end{cases}
$$

where $q \in(0,1), 2<\alpha \leq 3, \gamma \geq 0, \beta>0$, and $\lambda$ is a parameter, $D_{q}^{\alpha}$ is the $q$-derivative of Riemann-Liouville type of order $\alpha, f:[0,1] \times \mathbf{R} \rightarrow \mathbf{R}$ is continuous. In the present work, we gave the corresponding Green's function of the boundary value problem (1.1) and its properties. By using the generalized Banach contraction principle and Krasnoselskii's fixed-point theorem, the uniqueness, existence, and multiplicity of positive solution 
to the BVP (1.1) are obtained in term of the explicit intervals for the nonhomogeneous term. Our results are different from those of $[25,27]$.

\section{Preliminaries on $q$-calculus and lemmas}

For the convenience of the reader, below we cite some definitions and fundamental results on $q$-calculus as well as the fractional $q$-calculus. The presentation here can be found in, for example, $[12,18,20,22]$.

Let $q \in(0,1)$ and define

$$
[a]_{q}=\frac{1-q^{a}}{1-q}, \quad a \in \mathbf{R}
$$

The $q$-analogue of the power function $(a-b)^{n}$ with $n \in \mathbf{N}_{0}:=\{0,1,2, \ldots\}$ is

$$
(a-b)^{(0)}=1, \quad(a-b)^{(n)}=\prod_{k=0}^{n-1}\left(a-b q^{k}\right), \quad n \in \mathbf{N}, a, b \in \mathbf{R} .
$$

More generally, if $\alpha \in \mathbf{R}$, then

$$
(a-b)^{(\alpha)}=a^{\alpha} \prod_{k=0}^{\infty} \frac{a-b q^{k}}{a-b q^{\alpha+k}}, \quad a \neq 0 .
$$

Clearly, if $b=0$, then $a^{(\alpha)}=a^{\alpha}$. The $q$-gamma function is defined by

$$
\Gamma_{q}(x)=\frac{(1-q)^{(x-1)}}{(1-q)^{x-1}}, \quad x \in \mathbf{R} \backslash\{0,-1,-2, \ldots\},
$$

and satisfies $\Gamma_{q}(x+1)=[x]_{q} \Gamma_{q}(x)$.

The $q$-derivative of a function $f$ is defined by

$$
\left(D_{q} f\right)(x)=\frac{f(q x)-f(x)}{(q-1) x}, \quad\left(D_{q} f\right)(0)=\lim _{x \rightarrow 0}\left(D_{q} f\right)(x),
$$

and $q$-derivatives of higher order by

$$
\left(D_{q}^{0} f\right)(x)=f(x), \quad\left(D_{q}^{n} f\right)(x)=D_{q}\left(D_{q}^{n-1} f\right)(x), \quad n \in \mathbf{N}
$$

The $q$-integral of a function $f$ defined in the interval $[0, b]$ is given by

$$
\left(I_{q} f\right)(x)=\int_{0}^{x} f(s) d_{q} s=x(1-q) \sum_{k=0}^{\infty} f\left(x q^{k}\right) q^{k}, \quad x \in[0, b] .
$$

If $a \in[0, b]$ and $f$ is defined in the interval $[0, b]$, then its integral from $a$ to $b$ is defined by

$$
\int_{a}^{b} f(s) d_{q} s=\int_{0}^{b} f(s) d_{q} s-\int_{0}^{a} f(s) d_{q} s .
$$

Similar to that for derivatives, an operator $I_{q}^{n}$ is given by

$$
\left(I_{q}^{0} f\right)(x)=f(x), \quad\left(I_{q}^{n} f\right)(x)=I_{q}\left(I_{q}^{n-1} f\right)(x), \quad n \in \mathbf{N} .
$$


The fundamental theorem of calculus applies to these operators $I_{q}$ and $D_{q}$, i.e.,

$$
\left(D_{q} I_{q} f\right)(x)=f(x)
$$

and if $f$ is continuous at $x=0$, then

$$
\left(I_{q} D_{q} f\right)(x)=f(x)-f(0) .
$$

The following formulas will be used later, namely, the integration by parts formula:

$$
\int_{0}^{x} f(s)\left(D_{q} g\right)(s) d_{q} s=[f(s) g(s)]_{s=0}^{s=x}-\int_{0}^{x}\left(D_{q} f\right)(s) g(q s) d_{q} s,
$$

and

$$
\begin{aligned}
& {[a(t-s)]^{(\alpha)}=a^{\alpha}(t-s)^{(\alpha)},} \\
& { }_{t} D_{q}(t-s)^{(\alpha)}=[\alpha]_{q}(t-s)^{(\alpha-1)}, \\
& { }_{s} D_{q}(t-s)^{(\alpha)}=-[\alpha]_{q}(t-q s)^{(\alpha-1)}, \\
& \left({ }_{x} D_{q} \int_{0}^{x} f(x, s) d_{q} s\right)(x)=\int_{0}^{x}{ }_{x} D_{q} f(x, s) d_{q} s+f(q x, x),
\end{aligned}
$$

where ${ }_{t} D_{q}$ denotes the derivative with respect to the variable $t$.

Definition 2.1 Let $\alpha \geq 0$ and $f$ be a function defined on $[0,1]$. The fractional $q$-integral of Riemann-Liouville type is $\left(I_{q}^{0} f\right)(x)=f(x)$ and

$$
\left(I_{q}^{\alpha} f\right)(x)=\frac{1}{\Gamma_{q}(\alpha)} \int_{0}^{x}(x-q s)^{(\alpha-1)} f(s) d_{q} s, \quad \alpha>0, x \in[0,1] .
$$

Definition 2.2 The fractional $q$-derivative of the Riemann-Liouville type of order $\alpha \geq 0$ is defined by $\left(D_{q}^{0} f\right)(x)=f(x)$ and

$$
\left(D_{q}^{\alpha} f\right)(x)=\left(D_{q}^{[\alpha]} I_{q}^{[\alpha]-\alpha} f\right)(x), \quad \alpha>0,
$$

where $[\alpha]$ is the smallest integer greater than or equal to $\alpha$.

Lemma 2.3 ([20]) Assume that $\alpha \geq 0$ and $a \leq b \leq t$, then $(t-a)^{(\alpha)} \geq(t-b)^{(\alpha)}$.

Lemma 2.4 Let $\alpha, \beta \geq 0$ and $f$ be a function defined on $[0,1]$. Then the following formulas hold:

(1) $\left(I_{q}^{\beta} I_{q}^{\alpha} f\right)(x)=\left(I_{q}^{\alpha+\beta} f\right)(x)$,

(2) $\left(D_{q}^{\alpha} I_{q}^{\alpha} f\right)(x)=f(x)$.

Lemma 2.5 ([20]) Let $\alpha>0$ and $n$ be a positive integer. Then the following equality holds:

$$
\left(I_{q}^{\alpha} D_{q}^{n} f\right)(x)=\left(D_{q}^{n} I_{q}^{\alpha} f\right)(x)-\sum_{k=0}^{n-1} \frac{x^{\alpha-n+k}}{\Gamma_{q}(\alpha+k-n+1)}\left(D_{q}^{k} f\right)(0) .
$$


Lemma 2.6 ([22]) Let $\alpha \in \mathbf{R}^{+}, \lambda \in(-1,+\infty)$, the following is valid:

$$
I_{q}^{\alpha}\left((t-a)^{(\lambda)}\right)=\frac{\Gamma_{q}(\lambda+1)}{\Gamma_{q}(\alpha+\lambda+1)}(t-a)^{(\alpha+\lambda)}, \quad 0<a<t<b .
$$

Particularly, for $\lambda=0, a=0$, using $q$-integration by parts, we have

$$
\begin{aligned}
\left(I_{q}^{\alpha} 1\right)(t) & =\frac{1}{\Gamma_{q}(\alpha)} \int_{0}^{t}(t-q s)^{(\alpha-1)} d_{q} s=\frac{1}{\Gamma_{q}(\alpha)} \int_{0}^{t} \frac{{ }_{0} D_{q}\left((t-s)^{(\alpha)}\right)}{-[\alpha]_{q}} d_{q} s \\
& =-\frac{1}{\Gamma_{q}(\alpha+1)} \int_{0}^{t}{ }_{s} D_{q}\left((t-s)^{(\alpha)}\right) d_{q} s=\frac{1}{\Gamma_{q}(\alpha+1)} t^{(\alpha)} .
\end{aligned}
$$

Obviously, we have $\int_{0}^{t}(t-q s)^{(\alpha-1)} d_{q} s=\frac{1}{[\alpha]_{q}} t^{(\alpha)}$, and

$$
\begin{aligned}
\int_{0}^{t}(1-q s)^{(\alpha-1)} d_{q} s & =\int_{0}^{t} \frac{{ }_{s} D_{q}\left((1-s)^{(\alpha)}\right)}{-[\alpha]_{q}} d_{q} s \\
& =-\frac{1}{[\alpha]_{q}} \int_{0}^{t}{ }_{s} D_{q}\left((1-s)^{(\alpha)}\right) d_{q} s=\frac{1}{[\alpha]_{q}}\left[1-(1-t)^{(\alpha)}\right] .
\end{aligned}
$$

In order to define the solution for the problem (1.1), we need the following lemmas.

Lemma 2.7 For given $y \in C[0,1]$, the unique solution of the boundary value problem

$$
\left(D_{q}^{\alpha} u\right)(t)+y(t)=0, \quad t \in(0,1), 2<\alpha \leq 3,
$$

subject to the boundary conditions

$$
u(0)=\left(D_{q} u\right)(0)=0, \quad \gamma\left(D_{q} u\right)(1)+\beta\left(D_{q}^{2} u\right)(1)=\lambda,
$$

is given by

$$
u(t)=\int_{0}^{1} G(t, q s) y(s) d_{q} s+\frac{\lambda t^{\alpha-1}}{\left(\gamma+[\alpha-2]_{q} \beta\right)[\alpha-1]_{q}},
$$

where

$$
G(t, s)= \begin{cases}\frac{\gamma t^{\alpha-1}(1-s)^{(\alpha-2)}+[\alpha-2]_{q} \beta t^{\alpha-1}(1-s)^{(\alpha-3)}}{\left(\gamma+[\alpha-2]_{q} \beta\right) \Gamma_{q}(\alpha)}-\frac{(t-s)^{(\alpha-1)}}{\Gamma_{q}(\alpha)}, \quad 0 \leq s \leq t \leq 1, \\ \frac{\gamma t^{\alpha-1}(1-s)^{(\alpha-2)}+[\alpha-2]_{q} \beta t^{-\alpha}(1-s)^{(\alpha-3)}}{\left(\gamma+[\alpha-2]_{q} \beta\right) \Gamma_{q}(\alpha)}, \quad 0 \leq t \leq s \leq 1 .\end{cases}
$$

Proof Since $2<\alpha \leq 3$, we put $n=3$. In view of Definition 2.1 and Lemma 2.4, we see that

$$
\left(D_{q}^{\alpha} u\right)(t)=-y(t) \quad \Leftrightarrow \quad\left(I_{q}^{\alpha} D_{q}^{3} I_{q}^{3-\alpha} u\right)(t)=-\left(I_{q}^{\alpha} y\right)(t) .
$$

Then it follows from Lemma 2.5 that the solution $u(t)$ of (2.7) and (2.8) is given by

$$
u(t)=c_{1} t^{\alpha-1}+c_{2} t^{\alpha-2}+c_{3} t^{\alpha-3}-\int_{0}^{t} \frac{(t-q s)^{(\alpha-1)}}{\Gamma_{q}(\alpha)} y(s) d_{q} s,
$$

for some constants $c_{1}, c_{2}, c_{3} \in \mathbf{R}$. From $u(0)=0$, we have $c_{3}=0$. 
Differentiating both sides of (2.11) and with the help of (2.4) and (2.6), we obtain,

$$
\left(D_{q} u\right)(t)=[\alpha-1]_{q} c_{1} t^{\alpha-2}+[\alpha-2]_{q} c_{2} t^{\alpha-3}-\int_{0}^{t} \frac{[\alpha-1]_{q}(t-q s)^{(\alpha-2)}}{\Gamma_{q}(\alpha)} y(s) d_{q} s,
$$

and

$$
\begin{aligned}
\left(D_{q}^{2} u\right)(t)= & {[\alpha-1]_{q}[\alpha-2]_{q} c_{1} t^{\alpha-3}+[\alpha-2]_{q}[\alpha-3]_{q} c_{2} t^{\alpha-4} } \\
& -\int_{0}^{t} \frac{[\alpha-1]_{q}[\alpha-2]_{q}(t-q s)^{(\alpha-3)}}{\Gamma_{q}(\alpha)} y(s) d_{q} s .
\end{aligned}
$$

Then by the boundary condition $\left(D_{q} u\right)(0)=0$, we get $c_{2}=0$. Using the boundary condition $\gamma\left(D_{q} u\right)(1)+\beta\left(D_{q}^{2} u\right)(1)=\lambda$, we get

$$
\begin{aligned}
c_{1}= & \frac{1}{\gamma+[\alpha-2]_{q} \beta} \\
& \times\left(\gamma \int_{0}^{1} \frac{(1-q s)^{(\alpha-2)}}{\Gamma_{q}(\alpha)} y(s) d_{q} s+\beta \int_{0}^{1} \frac{[\alpha-2]_{q}(1-q s)^{(\alpha-3)}}{\Gamma_{q}(\alpha)} y(s) d_{q} s+\frac{\lambda}{[\alpha-1]_{q}}\right) .
\end{aligned}
$$

Hence, we have

$$
\begin{aligned}
u(t)= & \frac{t^{\alpha-1}}{\gamma+[\alpha-2]_{q} \beta}\left(\gamma \int_{0}^{1} \frac{(1-q s)^{(\alpha-2)}}{\Gamma_{q}(\alpha)} y(s) d_{q} s\right. \\
& \left.+\beta \int_{0}^{1} \frac{[\alpha-2]_{q}(1-q s)^{(\alpha-3)}}{\Gamma_{q}(\alpha)} y(s) d_{q} s+\frac{\lambda}{[\alpha-1]_{q}}\right)-\int_{0}^{t} \frac{(t-q s)^{(\alpha-1)}}{\Gamma_{q}(\alpha)} y(s) d_{q} s \\
= & \int_{0}^{1} G(t, q s) y(s) d_{q} s+\frac{\lambda t^{\alpha-1}}{\left(\gamma+[\alpha-2]_{q} \beta\right)[\alpha-1]_{q}} .
\end{aligned}
$$

This completes the proof of the lemma.

Lemma 2.8 The function $G(t, s)$ defined by (2.10) satisfies the following conditions:

(i) $G(t, q s) \geq 0$, and $G(t, q s) \leq G(1, q s)$ for all $0 \leq t, s \leq 1$.

(ii) $G(t, q s) \geq t^{\alpha-1} G(1, q s)$ for all $0 \leq t, s \leq 1$.

Proof We start by defining the following two functions:

$$
\begin{aligned}
& g_{1}(t, s)=\frac{\gamma t^{\alpha-1}(1-s)^{(\alpha-2)}+[\alpha-2]_{q} \beta t^{\alpha-1}(1-s)^{(\alpha-3)}}{\left(\gamma+[\alpha-2]_{q} \beta\right) \Gamma_{q}(\alpha)}, \quad 0 \leq t \leq s \leq 1, \\
& g_{2}(t, s)=g_{1}(t, s)-\frac{(t-s)^{(\alpha-1)}}{\Gamma_{q}(\alpha)}, \quad 0 \leq s \leq t \leq 1 .
\end{aligned}
$$

Obviously, $g_{1}(t, q s) \geq 0$. Now $g_{2}(0, q s)=0$, and for $t \neq 0$

$$
\begin{aligned}
g_{2}(t, q s) & =\frac{\gamma t^{\alpha-1}(1-q s)^{(\alpha-2)}+[\alpha-2]_{q} \beta t^{\alpha-1}(1-q s)^{(\alpha-3)}}{\left(\gamma+[\alpha-2]_{q} \beta\right) \Gamma_{q}(\alpha)}-\frac{(t-q s)^{(\alpha-1)}}{\Gamma_{q}(\alpha)} \\
& =\frac{t^{\alpha-1}\left(\gamma(1-q s)^{(\alpha-2)}+[\alpha-2]_{q} \beta(1-q s)^{(\alpha-3)}-\left(\gamma+[\alpha-2]_{q} \beta\right)\left(1-q_{t}^{s}\right)^{(\alpha-1)}\right)}{\left(\gamma+[\alpha-2]_{q} \beta\right) \Gamma_{q}(\alpha)}
\end{aligned}
$$




$$
\begin{aligned}
& \geq \frac{t^{\alpha-1}\left(\gamma(1-q s)^{(\alpha-2)}+[\alpha-2]_{q} \beta(1-q s)^{(\alpha-3)}-\left(\gamma+[\alpha-2]_{q} \beta\right)(1-q s)^{(\alpha-1)}\right)}{\left(\gamma+[\alpha-2]_{q} \beta\right) \Gamma_{q}(\alpha)} \\
& =\frac{t^{\alpha-1}\left(\gamma\left((1-q s)^{(\alpha-2)}-(1-q s)^{(\alpha-1)}\right)+[\alpha-2]_{q} \beta\left((1-q s)^{(\alpha-3)}-(1-q s)^{(\alpha-1)}\right)\right)}{\left(\gamma+[\alpha-2]_{q} \beta\right) \Gamma_{q}(\alpha)} \\
& \geq 0 .
\end{aligned}
$$

Therefore, $G(t, q s) \geq 0$.

Moreover, for $s \in(0,1]$, it follows from (2.4) and Lemma 2.3 that

$$
\begin{aligned}
{ }_{t} D_{q} g_{2}(t, q s)= & \frac{[\alpha-1]_{q}}{\left(\gamma+[\alpha-2]_{q} \beta\right) \Gamma_{q}(\alpha)}\left(\gamma t^{\alpha-2}(1-q s)^{(\alpha-2)}+[\alpha-2]_{q} \beta t^{\alpha-2}(1-q s)^{(\alpha-3)}\right. \\
& \left.-\left(\gamma+[\alpha-2]_{q} \beta\right)(t-q s)^{(\alpha-2)}\right) \\
\geq & \frac{[\alpha-1]_{q} t^{\alpha-2}}{\left(\gamma+[\alpha-2]_{q} \beta\right) \Gamma_{q}(\alpha)}\left(\gamma(1-q s)^{(\alpha-2)}+[\alpha-2]_{q} \beta(1-q s)^{(\alpha-3)}\right. \\
& \left.-\left(\gamma+[\alpha-2]_{q} \beta\right)(1-q s)^{(\alpha-2)}\right) \\
= & \frac{[\alpha-1]_{q}[\alpha-2]_{q} \beta t^{\alpha-2}}{\left(\gamma+[\alpha-2]_{q} \beta\right) \Gamma_{q}(\alpha)}\left((1-q s)^{(\alpha-3)}-(1-q s)^{(\alpha-2)}\right) \geq 0,
\end{aligned}
$$

which implies that $g_{2}(t, q s)$ is an increasing function with respect to $t$. It is clear that $g_{1}(t, q s)$ is increasing in $t$. Therefore, $G(t, q s)$ is an increasing function of $t$ for all $s \in(0,1]$, and so $G(t, q s) \leq G(1, q s)$.

When $0 \leq t \leq q s \leq 1$, then

$$
G(t, q s)=\frac{\gamma t^{\alpha-1}(1-q s)^{(\alpha-2)}+[\alpha-2]_{q} \beta t^{\alpha-1}(1-q s)^{(\alpha-3)}}{\left(\gamma+[\alpha-2]_{q} \beta\right) \Gamma_{q}(\alpha)} \leq G(q s, q s) \leq G(1, q s) .
$$

Finally, we prove part (ii). When $0 \leq q s \leq t \leq 1$, we have

$$
\begin{aligned}
& \frac{G(t, q s)}{G(1, q s)} \\
& \quad=\frac{\gamma t^{\alpha-1}(1-q s)^{(\alpha-2)}+[\alpha-2]_{q} \beta t^{\alpha-1}(1-q s)^{(\alpha-3)}-\left(\gamma+[\alpha-2]_{q} \beta\right)(t-q s)^{(\alpha-1)}}{\gamma(1-q s)^{(\alpha-2)}+[\alpha-2]_{q} \beta(1-q s)^{(\alpha-3)}-\left(\gamma+[\alpha-2]_{q} \beta\right)(1-q s)^{(\alpha-1)}} \\
& \quad \geq \frac{\gamma t^{\alpha-1}(1-q s)^{(\alpha-2)}+[\alpha-2]_{q} \beta t^{\alpha-1}(1-q s)^{(\alpha-3)}-\left(\gamma+[\alpha-2]_{q} \beta\right) t^{\alpha-1}(1-q s)^{(\alpha-1)}}{\gamma(1-q s)^{(\alpha-2)}+[\alpha-2]_{q} \beta(1-q s)^{(\alpha-3)}-\left(\gamma+[\alpha-2]_{q} \beta\right)(1-q s)^{(\alpha-1)}} \\
& \quad=t^{\alpha-1} .
\end{aligned}
$$

If $0 \leq t \leq q s \leq 1$, then we have

$$
\begin{aligned}
\frac{G(t, q s)}{G(1, q s)} & =\frac{\gamma t^{\alpha-1}(1-q s)^{(\alpha-2)}+[\alpha-2]_{q} \beta t^{\alpha-1}(1-q s)^{(\alpha-3)}}{\gamma(1-q s)^{(\alpha-2)}+[\alpha-2]_{q} \beta(1-q s)^{(\alpha-3)}-\left(\gamma+[\alpha-2]_{q} \beta\right)(1-q s)^{(\alpha-1)}} \\
& \geq \frac{t^{\alpha-1}\left[\gamma(1-q s)^{(\alpha-2)}+[\alpha-2]_{q} \beta(1-q s)^{(\alpha-3)}-\left(\gamma+[\alpha-2]_{q} \beta\right)(1-q s)^{(\alpha-1)}\right]}{\gamma(1-q s)^{(\alpha-2)}+[\alpha-2]_{q} \beta(1-q s)^{(\alpha-3)}-\left(\gamma+[\alpha-2]_{q} \beta\right)(1-q s)^{(\alpha-1)}} \\
& =t^{\alpha-1},
\end{aligned}
$$

which implies that part (ii) holds. This completes the proof of the lemma. 
Remark 2.9 If we let $0<\tau<1$, then

$$
\min _{t \in[\tau, 1]} G(t, q s) \geq \tau^{\alpha-1} G(1, q s), \quad \text { for } s \in[0,1]
$$

According to [20], we may take $\tau=q^{n}, n \in \mathbf{N}$.

\section{The main results}

Let $X=C([0,1])$ be a Banach space endowed with the norm $\|u\|_{X}=\max _{0 \leq t \leq 1}|u(t)|$. Define the cone $P \subset X$ by $P=\{u \in X: u(t) \geq 0,0 \leq t \leq 1\}$.

Define the operator $T: P \rightarrow X$ as follows:

$$
(T u)(t)=\int_{0}^{1} G(t, q s) f(s, u(s)) d_{q} s+\frac{\lambda t^{\alpha-1}}{\left(\gamma+[\alpha-2]_{q} \beta\right)[\alpha-1]_{q}} .
$$

Theorem 3.1 Assume that $f:[0,1] \times[0,+\infty) \rightarrow[0,+\infty)$ is continuous and there exists a nonnegative function $h \in C[0,1]$ such that

$$
|f(t, u)-f(t, v)| \leq h(t)|u-v|, \quad t \in[0,1], u, v \in[0,+\infty) .
$$

Then the BVP (1.1) has a unique positive solution for any $\lambda \in(0,+\infty)$, provided

$$
\int_{0}^{1} s^{\alpha-1}\left(\gamma(1-q s)^{(\alpha-2)}+[\alpha-2]_{q} \beta(1-q s)^{(\alpha-3)}\right) h(s) d_{q} s<\frac{\left(\gamma+[\alpha-2]_{q} \beta\right) \Gamma_{q}(\alpha)}{2} .
$$

If, in addition, $f(t, 0) \not \equiv 0$ on $[0,1]$, then the conclusion is true for $\lambda=0$.

Proof We will show that under the assumptions (3.2) and (3.3), $T^{m}$ is a contraction operator for $m$ sufficiently large.

By the definition of $G(t, q s)$, for $u, v \in P$, we have

$$
\begin{aligned}
& |(T u)(t)-(T v)(t)| \\
& \quad \leq \int_{0}^{1} G(t, q s)|f(s, u(s))-f(s, v(s))| d_{q} s \\
& \quad \leq \int_{0}^{1} \frac{t^{\alpha-1}\left(\gamma(1-q s)^{(\alpha-2)}+[\alpha-2]_{q} \beta(1-q s)^{(\alpha-3)}\right)}{\left(\gamma+[\alpha-2]_{q} \beta\right) \Gamma_{q}(\alpha)} h(s) d_{q} s \cdot\|u-v\|_{X} \\
& =\frac{t^{\alpha-1}\|u-v\|_{X}}{\left(\gamma+[\alpha-2]_{q} \beta\right) \Gamma_{q}(\alpha)} \int_{0}^{1}\left(\gamma(1-q s)^{(\alpha-2)}+[\alpha-2]_{q} \beta(1-q s)^{(\alpha-3)}\right) h(s) d_{q} s \\
& =\frac{\Lambda_{1} t^{\alpha-1}}{\left(\gamma+[\alpha-2]_{q} \beta\right) \Gamma_{q}(\alpha)}\|u-v\|_{X},
\end{aligned}
$$

where $\Lambda_{1}=\int_{0}^{1}\left(\gamma(1-q s)^{(\alpha-2)}+[\alpha-2]_{q} \beta(1-q s)^{(\alpha-3)}\right) h(s) d_{q} s$.

Consequently,

$$
\begin{aligned}
\left|\left(T^{2} u\right)(t)-\left(T^{2} v\right)(t)\right| & \leq \int_{0}^{1} G(t, q s)|f(s,(T u)(s))-f(s,(T v)(s))| d_{q} s \\
& \leq \frac{\Lambda_{1}\|u-v\|_{X}}{\left(\gamma+[\alpha-2]_{q} \beta\right) \Gamma_{q}(\alpha)} \int_{0}^{1} G(t, q s) s^{\alpha-1} h(s) d_{q} s
\end{aligned}
$$




$$
\begin{aligned}
\leq & \frac{\Lambda_{1} t^{\alpha-1}\|u-v\|_{X}}{\left(\gamma+[\alpha-2]_{q} \beta\right)^{2}\left[\Gamma_{q}(\alpha)\right]^{2}} \int_{0}^{1} s^{\alpha-1}\left(\gamma(1-q s)^{(\alpha-2)}\right. \\
& \left.+[\alpha-2]_{q} \beta(1-q s)^{(\alpha-3)}\right) h(s) d_{q} s \\
= & \frac{\Lambda_{1} \Lambda_{2} t^{\alpha-1}}{\left(\gamma+[\alpha-2]_{q} \beta\right)^{2}\left[\Gamma_{q}(\alpha)\right]^{2}}\|u-v\|_{X},
\end{aligned}
$$

where $\Lambda_{2}=\int_{0}^{1} s^{\alpha-1}\left(\gamma(1-q s)^{(\alpha-2)}+[\alpha-2]_{q} \beta(1-q s)^{(\alpha-3)}\right) h(s) d_{q} s$.

By introduction, we get

$$
\left|\left(T^{m} u\right)(t)-\left(T^{m} v\right)(t)\right| \leq \frac{\Lambda_{1} \Lambda_{2}^{m-1} t^{\alpha-1}}{\left(\gamma+[\alpha-2]_{q} \beta\right)^{m}\left[\Gamma_{q}(\alpha)\right]^{m}}\|u-v\|_{X} .
$$

From the condition (3.3), we have

$$
\begin{aligned}
\frac{\Lambda_{1} \Lambda_{2}^{m-1}}{\left(\gamma+[\alpha-2]_{q} \beta\right)^{m}\left[\Gamma_{q}(\alpha)\right]^{m}} & =\frac{\Lambda_{1}}{\Lambda_{2}}\left[\frac{\Lambda_{2}}{\left(\gamma+[\alpha-2]_{q} \beta\right) \Gamma_{q}(\alpha)}\right]^{m} \\
& \leq \frac{\Lambda_{1}}{\Lambda_{2}}\left(\frac{1}{2}\right)^{m} \\
& <\frac{1}{4},
\end{aligned}
$$

for $m$ sufficiently large. So, we get

$$
\left\|\left(T^{m} u\right)(t)-\left(T^{m} v\right)(t)\right\|_{X}<\frac{1}{4}\|u-v\|_{X} .
$$

Hence, it follows from the generalized Banach contraction principle that the BVP (1.1) has a unique positive solution for any $\lambda \in(0,+\infty)$. If $\lambda=0$, then the condition $f(t, 0) \not \equiv$ 0 on $[0,1]$ and Lemma 2.8 imply that $u(t)>0$ in $(0,1]$. This completes the proof of the theorem.

Remark 3.2 When $h(t) \equiv h_{0}$ is a constant, the condition (3.2) reduces to a Lipschitz condition.

Our next existence results is based on Krasnoselskii’s fixed-point theorem [33].

Lemma 3.3 (Krasnoselskii's) Let $E$ be a Banach space, and let $P \subset E$ be a cone. Assume $\Omega_{1}$, $\Omega_{2}$ are open subsets of $E$ with $\theta \in \Omega_{1}, \bar{\Omega}_{1} \subset \Omega_{2}$ and let $T: P \cap\left(\bar{\Omega}_{2} \backslash \Omega_{1}\right) \rightarrow P$ be a completely continuous operator such that, either

(1) $\|T u\| \geq\|u\|, u \in P \cap \partial \Omega_{1}$ and $\|T u\| \leq\|u\|, u \in P \cap \partial \Omega_{2}$, or

(2) $\|T u\| \leq\|u\|, u \in P \cap \partial \Omega_{1}$ and $\|T u\| \geq\|u\|, u \in P \cap \partial \Omega_{2}$.

Then $T$ has at least one fixed point in $P \cap\left(\bar{\Omega}_{2} \backslash \Omega_{1}\right)$.

Define a cone $K \subset X$ by

$$
K=\left\{u \in X: u(t) \geq 0, u(t) \geq t^{\alpha-1}\|u\|, t \in[0,1]\right\} .
$$

Obviously, $K$ is a cone of nonnegative functions in $X$. 
Lemma 3.4 The operator $T: K \rightarrow K$ is completely continuous.

Proof Firstly, we prove that $T(K) \subset K$. By (2.9) and Lemma 2.8, we have

$$
\begin{aligned}
\|T u\| & =\max _{0 \leq t \leq 1}\left\{\int_{0}^{1} G(t, q s) f(s, u(s)) d_{q} s+\frac{\lambda t^{\alpha-1}}{\left(\gamma+[\alpha-2]_{q} \beta\right)[\alpha-1]_{q}}\right\} \\
& \leq \int_{0}^{1} G(1, q s) f(s, u(s)) d_{q} s+\frac{\lambda}{\left(\gamma+[\alpha-2]_{q} \beta\right)[\alpha-1]_{q}} .
\end{aligned}
$$

On the other hand,

$$
(T u)(t) \geq t^{\alpha-1}\left(\int_{0}^{1} G(1, q s) f(s, u(s)) d_{q} s+\frac{\lambda}{\left(\gamma+[\alpha-2]_{q} \beta\right)[\alpha-1]_{q}}\right) \geq t^{\alpha-1}\|T u\| .
$$

Hence, we have $T(K) \subset K$.

Next, we show that $T$ is uniformly bounded. For fixed $r>0$, consider a bounded subset $K_{r}$ of $K$ defined by $K_{r}=\{u \in K:\|u\| \leq r, r>0\}$, and let $M=\max _{0 \leq u \leq r}|f(t, u)|+1$. Then for $u \in K_{r}$, we get

$$
\begin{aligned}
|(T u)(t)| & \leq \int_{0}^{1} G(t, q s)|f(s, u(s))| d_{q} s+\frac{|\lambda| t^{\alpha-1}}{\left(\gamma+[\alpha-2]_{q} \beta\right)[\alpha-1]_{q}} \\
& \leq M \int_{0}^{1} G(1, q s) d_{q} s+\frac{|\lambda|}{\left(\gamma+[\alpha-2]_{q} \beta\right)[\alpha-1]_{q}}<+\infty,
\end{aligned}
$$

which implies that $T\left(K_{r}\right)$ is bounded.

Finally, we show that $T$ is equicontinuous. For all $\varepsilon>0$, setting

$$
\delta=\min \left\{\frac{\varepsilon}{\omega(\alpha-1)}, \frac{1}{2}\left(\frac{\varepsilon}{\omega}\right)^{\frac{1}{\alpha-1}}\right\}
$$

where

$$
\omega=\frac{M\left(\gamma+[\alpha-2]_{q} \beta\right)+|\lambda| \Gamma_{q}(\alpha)}{\left(\gamma+[\alpha-2]_{q} \beta\right) \Gamma_{q}(\alpha)[\alpha-1]_{q}} .
$$

For any $u \in K_{r}$, we can prove that if $t_{1}, t_{2} \in[0,1]$ and $0<t_{2}-t_{1}<\delta$, then

$$
\left|(T u)\left(t_{2}\right)-(T u)\left(t_{2}\right)\right|<\varepsilon
$$

In fact, we have

$$
\begin{aligned}
& \left|(T u)\left(t_{2}\right)-(T u)\left(t_{1}\right)\right| \\
& \quad \leq \int_{0}^{1}\left|G\left(t_{2}, q s\right)-G\left(t_{1}, q s\right)\right| f(s, u(s)) d_{q} s+\frac{|\lambda|\left(t_{2}^{\alpha-1}-t_{1}^{\alpha-1}\right)}{\left(\gamma+[\alpha-2]_{q} \beta\right)[\alpha-1]_{q}} \\
& \quad \leq M \int_{0}^{1}\left|G\left(t_{2}, q s\right)-G\left(t_{1}, q s\right)\right| d_{q} s+\frac{|\lambda|\left(t_{2}^{\alpha-1}-t_{1}^{\alpha-1}\right)}{\left(\gamma+[\alpha-2]_{q} \beta\right)[\alpha-1]_{q}} \\
& \quad \leq M\left(\int_{0}^{t_{1}}\left|G\left(t_{2}, q s\right)-G\left(t_{1}, q s\right)\right| d_{q} s+\int_{t_{1}}^{t_{2}}\left|G\left(t_{2}, q s\right)-G\left(t_{1}, q s\right)\right| d_{q} s\right.
\end{aligned}
$$




$$
\begin{aligned}
& \left.+\int_{t_{2}}^{1}\left|G\left(t_{2}, q s\right)-G\left(t_{1}, q s\right)\right| d_{q} s\right)+\frac{|\lambda|\left(t_{2}^{\alpha-1}-t_{1}^{\alpha-1}\right)}{\left(\gamma+[\alpha-2]_{q} \beta\right)[\alpha-1]_{q}} \\
\leq & \frac{M}{\gamma+[\alpha-2]_{q} \beta}\left(\int_{0}^{t_{1}} \frac{\gamma(1-q s)^{(\alpha-2)}+[\alpha-2]_{q} \beta(1-q s)^{(\alpha-3)}}{\Gamma_{q}(\alpha)}\left(t_{2}^{\alpha-1}-t_{1}^{\alpha-1}\right) d_{q} s\right. \\
& +\int_{t_{1}}^{t_{2}} \frac{\gamma(1-q s)^{(\alpha-2)}+[\alpha-2]_{q} \beta(1-q s)^{(\alpha-3)}}{\Gamma_{q}(\alpha)}\left(t_{2}^{\alpha-1}-t_{1}^{\alpha-1}\right) d_{q} s \\
& \left.+\int_{t_{2}}^{1} \frac{\gamma(1-q s)^{(\alpha-2)}+[\alpha-2]_{q} \beta(1-q s)^{(\alpha-3)}}{\Gamma_{q}(\alpha)}\left(t_{2}^{\alpha-1}-t_{1}^{\alpha-1}\right) d_{q} s\right) \\
& +\frac{|\lambda|\left(t_{2}^{\alpha-1}-t_{1}^{\alpha-1}\right)}{\left(\gamma+[\alpha-2]_{q} \beta\right)[\alpha-1]_{q}} \\
= & \frac{M\left(t_{2}^{\alpha-1}-t_{1}^{\alpha-1}\right)}{\left(\gamma+[\alpha-2]_{q} \beta\right) \Gamma_{q}(\alpha)} \int_{0}^{1}\left(\gamma(1-q s)^{(\alpha-2)}+[\alpha-2]_{q} \beta(1-q s)^{(\alpha-3)}\right) d_{q} s \\
& +\frac{|\lambda|}{\left(\gamma+[\alpha-2]_{q} \beta\right)[\alpha-1]_{q}}\left(t_{2}^{\alpha-1}-t_{1}^{\alpha-1}\right) \\
= & \frac{M\left(\gamma+[\alpha-2]_{q} \beta\right)+|\lambda| \Gamma_{q}(\alpha)}{\left(\gamma+[\alpha-2]_{q} \beta\right) \Gamma_{q}(\alpha)[\alpha-1]_{q}}\left(t_{2}^{\alpha-1}-t_{1}^{\alpha-1}\right) \\
= & \omega\left(t_{2}^{\alpha-1}-t_{1}^{\alpha-1}\right) .
\end{aligned}
$$

If $\delta \leq t_{1}<t_{2}<1$, then

$$
\left|(T u)\left(t_{2}\right)-(T u)\left(t_{1}\right)\right| \leq \omega\left(t_{2}^{\alpha-1}-t_{1}^{\alpha-1}\right)<\omega(\alpha-1)\left(t_{2}-t_{1}\right)<\omega(\alpha-1) \delta \leq \varepsilon .
$$

If $0 \leq t_{1}<\delta, t_{2}<2 \delta$, then

$$
\left|(T u)\left(t_{2}\right)-(T u)\left(t_{1}\right)\right| \leq \omega\left(t_{2}^{\alpha-1}-t_{1}^{\alpha-1}\right)<\omega t_{2}^{\alpha-1}<\omega(2 \delta)^{\alpha-1} \leq \varepsilon
$$

By means of Arzela-Ascoli theorem, $T: K \rightarrow K$ is completely continuous.

For the sake of convenience, we introduce the following weight functions:

$$
\begin{aligned}
& \phi(r)=\max \{f(t, u(t)):(t, u) \in[0,1] \times[0, r]\}, \\
& \varphi(r)=\min \left\{f(t, u(t)):(t, u) \in[\tau, 1] \times\left[\tau^{\alpha-1} r, r\right]\right\},
\end{aligned}
$$

and set

$$
l=\left(\int_{0}^{1} G(1, q s) d_{q} s\right)^{-1}, \quad L=\left(\tau^{\alpha-1} \int_{\tau}^{1} G(1, q s) d_{q} s\right)^{-1} .
$$

Theorem 3.5 Suppose that there exists two positive numbers $\xi_{1}<\xi_{2}$ such that one of the following conditions is satisfied

$\left(H_{1}\right) \varphi\left(\xi_{1}\right) \geq \xi_{1} L, \phi\left(\xi_{2}\right) \leq \frac{l}{2} \xi_{2} ;$

$\left(H_{2}\right) \phi\left(\xi_{1}\right) \leq \frac{l}{2} \xi_{1}, \varphi\left(\xi_{2}\right) \geq \xi_{2} L$.

Then the BVP (1.1) has at least one positive solution $u^{*} \in K$, such that $\xi_{1} \leq\left\|u^{*}\right\| \leq \xi_{2}$ for $\lambda \in\left(0, \frac{\left(\gamma+[\alpha-2]_{q} \beta\right)[\alpha-1]_{q}}{2} \xi_{1}\right]$. If, in addition, $f(t, 0) \not \equiv 0$ on $[0,1]$, then the conclusion is true for $\lambda=0$. 
Proof Because the proofs are similar, we prove only the case $\left(H_{1}\right)$. Denote $\Omega_{\xi_{1}}=\{u \in X$ : $\left.\|u\|<\xi_{1}\right\}$. Then for any $u \in K \cap \partial \Omega_{\xi_{1}}$, we get $\|u\|=\xi_{1}, 0 \leq u(t) \leq \xi_{1}, 0 \leq t \leq 1$, and $\tau^{\alpha-1} \xi_{1} \leq$ $\min _{\tau \leq t \leq 1} t^{\alpha-1}\|u\| \leq u(t) \leq\|u\|=\xi_{1}, \tau \leq t \leq 1$. By assumption $\left(H_{1}\right)$, we have

$$
f(t, u) \geq \varphi\left(\xi_{1}\right) \geq \xi_{1} L, \quad \tau \leq t \leq 1 .
$$

In view of (2.9) and Lemma 2.8, we have

$$
\begin{aligned}
\|T u\| & \geq \min _{\tau \leq t \leq 1}\left\{\int_{0}^{1} G(t, q s) f(s, u(s)) d_{q} s+\frac{\lambda t^{\alpha-1}}{\left(\gamma+[\alpha-2]_{q} \beta\right)[\alpha-1]_{q}}\right\} \\
& \geq \min _{\tau \leq t \leq 1} \int_{0}^{1} G(t, q s) f(s, u(s)) d_{q} s \\
& \geq \tau^{\alpha-1} \int_{\tau}^{1} G(1, q s) f(s, u(s)) d_{q} s \\
& \geq \tau^{\alpha-1} \int_{\tau}^{1} G(1, q s) d_{q} s \cdot \xi_{1} L=\xi_{1}=\|u\| .
\end{aligned}
$$

On the other hand, define $\Omega_{\xi_{2}}=\left\{u \in X:\|u\|<\xi_{2}\right\}$. For any $t \in[0,1]$ and $u \in K \cap \partial \Omega_{\xi_{2}}$, we have $\|u\|=\xi_{2}$ and $0 \leq u(t) \leq \xi_{2}, 0 \leq t \leq 1$. Thus,

$$
f(t, u) \leq \phi\left(\xi_{2}\right) \leq \frac{l}{2} \xi_{2}, \quad \text { for } 0 \leq t \leq 1,0 \leq u \leq \xi_{2} .
$$

It follows

$$
\begin{aligned}
\|T u\| & =\max _{0 \leq t \leq 1}\left\{\int_{0}^{1} G(t, q s) f(s, u(s)) d_{q} s+\frac{\lambda t^{\alpha-1}}{\left(\gamma+[\alpha-2]_{q} \beta\right)[\alpha-1]_{q}}\right\} \\
& \leq \int_{0}^{1} G(1, q s) d_{q} s \cdot \frac{l}{2} \xi_{2}+\frac{\lambda}{\left(\gamma+[\alpha-2]_{q} \beta\right)[\alpha-1]_{q}} \\
& \leq l^{-1} \cdot \frac{l}{2} \xi_{2}+\frac{\xi_{1}}{2}<\frac{\xi_{2}}{2}+\frac{\xi_{2}}{2}=\|u\| .
\end{aligned}
$$

By Lemma 3.3, the operator $T$ has at least one fixed point $u^{*} \in K \cap\left(\bar{\Omega}_{\xi_{2}} \backslash \Omega_{\xi_{1}}\right)$, and $\xi_{1} \leq$ $\left\|u^{*}\right\| \leq \xi_{2}$. Since $u^{*}(t) \geq t^{\alpha-1}\left\|u^{*}\right\| \geq \xi_{1} t^{\alpha-1}>0,0<t<1$, then, the solution $u^{*}$ is positive for $\lambda>0$. As in the proof of Theorem 3.1, $u^{*}(t)$ is a positive solution for $\lambda=0$. This completes the proof of the theorem.

Theorem 3.6 Suppose that there exists three positive numbers $\xi_{1}<\xi_{2}<\xi_{3}$ such that one of the following conditions is satisfied

$\left(H_{3}\right) \varphi\left(\xi_{1}\right) \geq \xi_{1} L, \phi\left(\xi_{2}\right)<\frac{l}{2} \xi_{2}, \varphi\left(\xi_{3}\right) \geq \xi_{3} L$

$\left(H_{4}\right) \phi\left(\xi_{1}\right) \leq \frac{l}{2} \xi_{1}, \varphi\left(\xi_{2}\right)>\xi_{2} L, \phi\left(\xi_{3}\right) \leq \frac{l}{2} \xi_{3}$.

Then the BVP (1.1) has at least two positive solutions $u_{1}^{*}, u_{2}^{*} \in K$ such that $\xi_{1} \leq\left\|u_{1}^{*}\right\|<$ $\xi_{2} \leq\left\|u_{2}^{*}\right\| \leq \xi_{3}$ for $\lambda \in\left(0, \frac{\left(\gamma+[\alpha-2]_{q} \beta\right)[\alpha-1]_{q}}{2} \xi_{1}\right]$. If, in addition, $f(t, 0) \not \equiv 0$ on $[0,1]$, then the conclusion is true for $\lambda=0$.

Proof We prove only the case $\left(H_{4}\right)$. Since $\varphi:[0,+\infty) \rightarrow[0,+\infty)$ is continuous and $\varphi\left(\xi_{2}\right)>$ $\xi_{2} L$, there exist two positive numbers $\eta_{1}, \eta_{2}$ such that $\xi_{1}<\eta_{1}<\xi_{2}<\eta_{2}<\xi_{3}$ and $\varphi\left(\eta_{1}\right) \geq \eta_{1} L$, 
$\varphi\left(\eta_{2}\right) \geq \eta_{2} L$. Thus, it follows from the assumption $\left(H_{4}\right)$ that

$$
\phi\left(\xi_{1}\right) \leq \frac{l}{2} \xi_{1}, \quad \varphi\left(\eta_{1}\right) \geq \eta_{1} L, \quad \text { and } \quad \varphi\left(\eta_{2}\right) \geq \eta_{2} L, \quad \phi\left(\xi_{3}\right) \leq \frac{l}{2} \xi_{3} .
$$

From Theorem 3.5, the operator $T$ has two fixed point $u_{1}^{*} \in K \cap\left(\bar{\Omega}_{\eta_{1}} \backslash \Omega_{\xi_{1}}\right), u_{2}^{*} \in K \cap$ $\left(\bar{\Omega}_{\xi_{3}} \backslash \Omega_{\eta_{2}}\right)$ with $\xi_{1} \leq\left\|u_{1}^{*}\right\|<\xi_{2}<\left\|u_{2}^{*}\right\| \leq \xi_{3}$. Therefore, the BVP (1.1) has at least two positive solutions for $\lambda \in\left(0, \frac{\left(\gamma+[\alpha-2]_{q} \beta\right)[\alpha-1]_{q}}{2} \xi_{1}\right]$. As in the proof of Theorem 3.1, $u_{1}^{*}, u_{2}^{*}$ are two positive solutions for $\lambda=0$. This completes the proof of the theorem.

Denote the integer part of $m$ by $[m]$. Generally, we have the following theorem.

Theorem 3.7 Suppose that there exists $m+1$ positive numbers $\xi_{1}<\xi_{2}<\cdots<\xi_{m+1}$ such that one of the following conditions is satisfied:

$\left(H_{5}\right) \varphi\left(\xi_{2 j-1}\right)>\xi_{2 j-1} L, \phi\left(\xi_{2 j}\right)<\frac{l}{2} \xi_{2 j}, j=1,2, \ldots,\left[\frac{m+2}{2}\right]$;

( $\left.H_{6}\right) \phi\left(\xi_{2 j-1}\right)<\frac{l}{2} \xi_{2 j-1}, \varphi\left(\xi_{2 j}\right)>\xi_{2 j} L, j=1,2, \ldots,\left[\frac{m+2}{2}\right]$.

Then the BVP (1.1) has at least $m$ positive solutions $u_{i}^{*} \in K, i=1,2, \ldots, m$, such that $\xi_{i}<\left\|u_{i}^{*}\right\|<\xi_{i+1}$ for $\lambda \in\left(0, \frac{\left(\gamma+[\alpha-2]_{q} \beta\right)[\alpha-1]_{q}}{2} \xi_{1}\right]$. If, in addition, $f(t, 0) \not \equiv 0$ on $[0,1]$, then the conclusion is true for $\lambda=0$.

\section{Examples}

Example 4.1 The fractional $q$-difference boundary value problem

$$
\left\{\begin{array}{l}
D_{0.5}^{2.5} u(t)+\frac{2 e^{t}}{5\left(1+e^{t}\right)}\left(\tan ^{-1} u+t^{2}+t \sin ^{2} t+1\right)=0, \quad 0<t<1, \\
u(0)=\left(D_{0.5} u\right)(0)=0, \quad 0.25\left(D_{0.5} u\right)(1)+0.75\left(D_{0.5}^{2} u\right)(1)=\lambda
\end{array}\right.
$$

has a unique positive solution for any $\lambda \in(0,+\infty)$.

Proof In this case, $\alpha=2.5, q=0.5, \gamma=0.25, \beta=0.75, \lambda>0$. Let

$$
f(t, u)=\frac{2 e^{t}}{5\left(1+e^{t}\right)}\left(\tan ^{-1} u+t^{2}+t \sin ^{2} t+1\right), \quad(t, u) \in[0,1] \times(0,+\infty),
$$

and $h(t)=\frac{2 e^{t}}{5\left(1+e^{t}\right)}$. It is easy to prove that

$$
\begin{aligned}
|f(t, u)-f(t, v)| & \leq h(t)\left|\tan ^{-1} u-\tan ^{-1} v\right| \\
& \leq h(t)|u-v|, \quad \text { for }(t, u),(t, v) \in[0,1] \times[0,+\infty) .
\end{aligned}
$$

A simple computation showed

$$
\left(\gamma+[\alpha-2]_{q} \beta\right) \Gamma_{q}(\alpha)=\left(0.25+[0.5]_{0.5} \times 0.75\right) \Gamma_{0.5}(2.5) \approx 0.9168,
$$

and

$$
\begin{aligned}
\Lambda_{2} & =\int_{0}^{1} s^{\alpha-1}\left(\gamma(1-q s)^{(\alpha-2)}+[\alpha-2]_{q} \beta(1-q s)^{(\alpha-3)}\right) \cdot \frac{2 e^{s}}{5\left(1+e^{s}\right)} d_{q} s \\
& \leq \frac{2}{5} \int_{0}^{1} s^{\alpha-1}\left(\gamma(1-q s)^{(\alpha-2)}+[\alpha-2]_{q} \beta(1-q s)^{(\alpha-3)}\right) d_{q} s
\end{aligned}
$$




$$
\begin{aligned}
& \leq \frac{2}{5} \int_{0}^{1}\left(\gamma(1-q s)^{(\alpha-2)}+[\alpha-2]_{q} \beta(1-q s)^{(\alpha-3)}\right) d_{q} s \\
& \approx 0.3774
\end{aligned}
$$

which implies that

$$
\int_{0}^{1} s^{\alpha-1}\left(\gamma(1-q s)^{(\alpha-2)}+[\alpha-2]_{q} \beta(1-q s)^{(\alpha-3)}\right) h(s) d_{q} s<\frac{\left(\gamma+[\alpha-2]_{q} \beta\right) \Gamma_{q}(\alpha)}{2} .
$$

Obviously, for any $m \geq 2$, we have

$$
\frac{\Lambda_{1} \Lambda_{2}^{m-1}}{\left(\gamma+[\alpha-2]_{q} \beta\right)^{m}\left[\Gamma_{q}(\alpha)\right]^{m}} \leq \frac{0.3774}{0.9168 \times 2^{m-1}}<0.2058<\frac{1}{4}
$$

Thus, Theorem 3.1 implies that the boundary value problem (4.1) has a unique positive solution for any $\lambda \in(0,+\infty)$.

Example 4.2 Consider the following fractional boundary value problem:

$$
\left\{\begin{array}{l}
D_{0.5}^{2.5} u(t)+u^{2}\left(\frac{1}{2} \cos \left(\pi t-\frac{\pi}{2}\right)+\frac{1}{4}\right)=0, \quad 0<t<1 \\
u(0)=\left(D_{0.5} u\right)(0)=0, \quad 2\left(D_{0.5} u\right)(1)+7\left(D_{0.5}^{2} u\right)(1)=\lambda,
\end{array}\right.
$$

where $\alpha=2.5, q=0.5, \gamma=2, \beta=7$. Choosing $n=1$, then $\tau=q^{n}=0.5$.

By calculation, we get $\left(\gamma+[\alpha-2]_{q} \beta\right) \Gamma_{q}(\alpha) \approx 8.1132$. By Lemma 2.6, Lemma 2.8 and with the aid of a computer, we obtain that

$$
l=\left(\int_{0}^{1} G(1,0.5 s) d_{0.5} s\right)^{-1} \approx 1.6756
$$

and

$$
L=\left((0.5)^{\alpha-1} \int_{0.5}^{1} G(1,0.5 s) d_{0.5} s\right)^{-1} \approx 0.0657
$$

Let $f(t, u)=u^{2}\left(\frac{1}{2} \cos \left(\pi t-\frac{\pi}{2}\right)+\frac{1}{4}\right)$. Take $\xi_{1}=\frac{3}{4}, \xi_{2}=3$, then $\frac{\left(\gamma+[\alpha-2]_{q} \beta\right)[\alpha-1]_{q}}{2} \xi_{1} \approx 2.9578$, and $f(t, u)$ satisfies

(i) $\phi\left(\frac{3}{4}\right)=\max \left\{u^{2}\left(\frac{1}{2} \cos \left(\pi t-\frac{\pi}{2}\right)+\frac{1}{4}\right):(t, u) \in[0,1] \times\left[0, \frac{3}{4}\right]\right\}=\frac{27}{64}<\frac{l}{2} \xi_{1} \approx 0.6283$;

(ii) $\varphi(3)=\min \left\{u^{2}\left(\frac{1}{2} \cos \left(\pi t-\frac{\pi}{2}\right)+\frac{1}{4}\right):(t, u) \in[0.5,1] \times[1.0608,3]\right\} \approx 0.2652>L \xi_{2} \approx$ 0.1971 .

So, by Theorem 3.5 , the problem (4.2) has one positive solution $u^{*}$ such that $1 \leq\left\|u^{*}\right\|_{X} \leq$ 3 for $\lambda \in(0,2.9578]$. 


\section{Author details}

'School of Science, Hunan University of Technology, Zhuzhou, 412007, China. ${ }^{2}$ Department of Mathematics, Central South University, Changsha, 410075, China.

\section{Acknowledgements}

Dedicated to Professor Hari M Srivastava.

The authors are highly grateful for the referees' careful reading and comments on this paper. The research is supported by the National Natural Science Foundation of China (Grant No. 11271372, 11201138); it is also supported by the Hunan Provincial Natural Science Foundation of China (Grant No. 13JJ3106, 12JJ2004), and the Scientific Research Fund of Hunan Provincial Education Department (Grant No. 12B034).

\section{Received: 17 January 2013 Accepted: 12 April 2013 Published: 25 April 2013}

\section{References}

1. Podlubny, I: Fractional Differential Equations. Academic Press, San Diego (1999)

2. Kibas, AA, Srivastava, HM, Trujillo, JJ: Theory and Applications of Fractional Differential Equations. Elsevier, Amsterdam (2006)

3. Al-Refai, M, Ali Hajji, M: Monotone iterative sequences for nonlinear boundary value problems of fractional order. Nonlinear Anal. 74, 3531-3539 (2011)

4. Ahmad, B, Sivasundaram, S: On four-point nonlocal boundary value problems of nonlinear integro-differential equations of fractional order. Appl. Math. Comput. 217, 480-487 (2010)

5. Stanék, S: The existence of positive solutions of singular fractional boundary value problems. Comput. Math. Appl. 62, 1379-1388 (2011)

6. Zhao, Y, Chen, H, Huang, L: Existence of positive solutions for nonlinear fractional functional differential equation. Comput. Math. Appl. 64, 3456-3467 (2012)

7. Agarwal, RP, O'Regan, D, Stanek, S: Positive solutions for Dirichlet problems of singular nonlinear fractional differential equations. J. Math. Anal. Appl. 371, 57-68 (2010)

8. Goodrich, CS: On discrete sequential fractional boundary value problems. J. Math. Anal. Appl. 385, 111-124 (2012)

9. Zhang, S: Positive solutions to singular boundary value problem for nonlinear fractional differential equation. Comput. Math. Appl. 59, 1300-1309 (2010)

10. Zhang, X, Liu, L, Wu, Y: Multiple positive solutions of a singular fractional differential equation with negatively perturbed term. Math. Comput. Model. 55, 1263-1274 (2012)

11. Jackson, FH: q-Difference equations. Am. J. Math. 32, 305-314 (1970)

12. Kac, V, Cheung, P: Quantum Calculus. Springer, New York (2002)

13. Ernst, T: The history of $q$-calculus and a new method. UUDM Report 2000:16, Department of Mathematics, Uppsala University, 2000, ISSN:1101-3591

14. Ahmad, B: Boundary-value problems for nonlinear third-order q-difference equations. Electron. J. Differ. Equ. 2011, 94 (2011)

15. Goodrich, CS: Existence of a positive solution to a system of discrete fractional boundary value problems. Appl. Math Comput. 217, 4740-4753 (2011)

16. Al-Salam, WA: Some fractional q-integrals and q-derivatives. Proc. Edinb. Math. Soc. 15(2), 135-140 (1966/1967)

17. Agarwal, RP: Certain fractional $q$-integrals and q-derivatives. Proc. Camb. Philos. Soc. 66, 365-370 (1969)

18. Annaby, MH, Mansour, IS: q-Fractional Calculus and Equations. Springer, Berlin (2012)

19. Atici, FM, Eloe, PW: Fractional q-calculus on a time scale. J. Nonlinear Math. Phys. 14, 333-344 (2007)

20. Ferreira, RAC: Nontrivial solutions for fractional q-difference boundary value problems. Electron. J. Qual. Theory Differ. Equ. 70, 1-10 (2010)

21. Goodrich, CS: Continuity of solutions to discrete fractional initial value problem. Comput. Math. Appl. 59, 3489-3499 (2010)

22. Rajković, PM, Marinković, SD, Stanković, MS: Fractional integrals and derivatives in q-calculus. Appl. Anal. Discrete Math. 1, 311-323 (2007)

23. Atici, FM, Eloe, PW: Two-point boundary value problems for finite fractional difference equations. J. Differ. Equ. Appl. 17, 445-456 (2011)

24. Ferreira, RAC: Positive solutions for a class of boundary value problems with fractional $q$-differences. Comput. Math. Appl. 61, 367-373 (2011)

25. El-Shahed, M, Al-Askar, F: Positive solutions for boundary value problem of nonlinear fractional $q$-difference equation ISRN Math. Anal. 2011, Article ID 385459 (2011)

26. Liang, S, Zhang, J: Existence and uniqueness of positive solutions for three-point boundary value problem with fractional q-differences. J. Appl. Math. Comput. 40, 277-288 (2012)

27. Graef, JR, Kong, L: Positive solutions for a class of higher order boundary value problems with fractional $q$-derivatives. Appl. Math. Comput. 218, 9682-9689 (2012)

28. $\mathrm{Ma}$, J, Yang, J: Existence of solutions for multi-point boundary value problem of fractional $q$-difference equation Electron. J. Qual. Theory Differ. Equ. 92, 1-10 (2011)

29. Ahmad, B, Ntouyas, S, Purnaras, I: Existence results for nonlocal boundary value problems of nonlinear fractional q-difference equations. Adv. Differ. Equ. 2012, 140 (2012)

30. El-Shahed, M, Gaber, M: Two-dimensional q-differential transformation and its application. Appl. Math. Comput. 217, 9165-9172 (2011)

31. Zhao, Y, Chen, H, Zhang, Q: Existence results for fractional $q$-difference equations with nonlocal $q$-integral boundary conditions. Adv. Differ. Equ. 2013, 48 (2013)

32. Zhao, Y, Ye, G, Chen, $\mathrm{H}$ : Multiple positive solutions of a singular semipositone integral boundary value problem for fractional q-derivatives equation. Abstr. Appl. Anal. 2013, Article ID 643571 (2013)

33. Guo, DJ, Lakshmikantham, V: Nonlinear Problems in Abstract Cones. Academic Press, San Diego (1988) 
doi:10.1186/1687-2770-2013-103

Cite this article as: Zhao et al.: Existence and multiplicity of positive solutions for nonhomogeneous boundary value problems with fractional q-derivatives. Boundary Value Problems 2013 2013:103.

Submit your manuscript to a SpringerOpen ${ }^{\circ}$ journal and benefit from:

- Convenient online submission

- Rigorous peer review

- Immediate publication on acceptance

- Open access: articles freely available online

- High visibility within the field

- Retaining the copyright to your article

Submit your next manuscript at $\gg$ springeropen.com 